\title{
The Study of Mean-Variance Risky Asset Management with State- Dependent Risk Aversion under Regime Switching Market
}

\author{
Shuang Li $\mathbb{D}^{1},{ }^{1}$ Yu Yang $\mathbb{D}^{2},{ }^{2}$ Yanli Zhou $\mathbb{D}^{3},{ }^{3}$ Yonghong $\mathrm{Wu}^{2}$ and Xiangyu Ge $\mathbb{D}^{4}$ \\ ${ }^{1}$ Department of Mathematics and Physics, Mianyang Normal University, Mianyang 621000, China \\ ${ }^{2}$ Department of Mathematics and Statistics, Curtin University, Perth, WA 6102, Australia \\ ${ }^{3}$ School of Finance, Zhongnan University of Economics and Law, Wuhan 430073, China \\ ${ }^{4}$ School of Statistics and Mathematics, Zhongnan University of Economics and Law, Wuhan 430073, China
}

Correspondence should be addressed to Xiangyu Ge; xiangyu_ge@163.com

Received 20 August 2021; Accepted 5 November 2021; Published 20 November 2021

Academic Editor: Fanglei Wang

Copyright (C) 2021 Shuang Li et al. This is an open access article distributed under the Creative Commons Attribution License, which permits unrestricted use, distribution, and reproduction in any medium, provided the original work is properly cited.

\begin{abstract}
How do investors require a distribution of the wealth among multiple risky assets while facing the risk of the uncontrollable payment for random liabilities? To cope with this problem, firstly, this paper explores the approach of asset-liability management under the state-dependent risk aversion with only risky assets, which has been considered under a continuoustime Markov regime-switching setting. Next, based on this realistic modelling, an extended Hamilton-Jacob-Bellman (HJB) system has been necessarily established for solving the optimization problem of asset-liability management. It has been derived closed-form analytical expressions applied in the time-inconsistent investment with optimal control theory to see that happens to the optimal value of the function. Ultimately, numerical examples presented with comparisons of the analytical results under different market conditions are exposed to analyse numerically the developed mean variance asset liability management strategy. We find that our proposed model can explain the financial phenomena more effectively and accurately.
\end{abstract}

\section{Introduction}

Portfolio optimization selection problem, well known as an essential topic in financial markets, has been done in deep researches by many scholars after the first reported by Markowitz [1]. The most frequently used method of optimal asset allocation strategies is HJB equation, i.e., the Hamilton-Jacobi-Bellman equation (see Detemple and Fernando [2], Björk et al. [3]). In the analysis of portfolio optimization, utility function and several system parameters are given to find the optimal values of the control parameters to realise the final utility maximization. Previous researches in this area are classified for the endogenous habit formation [2], the classic constant relative risk aversion (CRRA) by Yu and Yuan [4], the hyperbolic discounting [3], and the utilities like the mean-variance utility proposed by $\mathrm{Li}$ et al. [5]. In recent paper by $\mathrm{Li}$ et al. [6], the analytical solution portfolio optimization problem involving stochastic shortterm interest rates is provided, which can be controlled by the mean-variance utility function with state dependent risk aversion (SDRA). The paper [6] uses the Nash equilibrium for the subgame strategy to concrete analytical expressions of value function and control policy of equilibrium and figure out under the condition of the stochastic short-term interest rates, how do investors with "natural risk aversion" achieve optimal control policies by simplifying financial settings.

Under the framework of mean variance equilibrium asset liability management with SDRA, some extended models have been constructed, such as the mean-variance asset-liability management problem by regime-switching models, as well as mean-variance models with only risky assets (see Bening and Koroley [7]; the asset-only models to asset-liability models have been greatly expanded by Yao et al. $[8,9])$. A geometric method raised by Leippold et al. [10] is supposed to apply into the multiperiod meanvariance asset-liability management model by taking the implied mean-variance of liability frontier into consideration. A study by Chiu and Li [11] reported that the influence of the rebalancing frequency is quantified to 
determine the allocation of optimal initial funds. The work of extension into a continuous-time setting has been developed with the aid of a stochastic linear quadratic control approach. Based on the assumptions used in Leippold et al. [10], analytical results have been derived in a complete market with discussing the impact of liability on the optimal funding ratio. To construct more realistic models, more focus has been put on studying the asset-liability management under the market behavior in the face of many restriction conditions, for example, an uncertain investment horizon (see $\mathrm{Li}$ and $\mathrm{Ng}$ [12]; Li and Yao [13]), regimeswitching to describe phenomena between "Bullish" and "Bearish" markets (see Elliott et al. [14]; Wei et al. [15]; $\mathrm{Wu}$ and $\mathrm{Li}$ [16]; $\mathrm{Wu}$ and Chen [17]; Yu [18]), the choice of optimal portfolio selection for assets with transaction costs without short sales (see Li et al. [19]), portfolio selection under partial information (see Xiong and Zhou [20]), bankruptcy control (see Li and Li [21]), jump-diffusion in financial markets (see Lim [22]; Zeng and Li [23]), and stochastic volatility and stochastic interest rates (see Lim [22]; Lim and Zhou [24]). Also, various studies of assets and liabilities management problem have been carried out in some particular field with application in insurance and pension fund, including Drijver [25] for pension funds Hilli et al. [26] for a Finnish pension company, and Gerstner et al. [27] and Chiu and Wong [28] for life insurance policies.

Among them, regime-switching models have become popular in finance and related fields, which is expected to describe the characteristics of different markets (called "Bullish" versus "Bearish"). A limited number of regimes have been applied to represent the various patterns of the market states. According to diverse financial markets as the change market pattern occurs, indices for instance the interest rate, appreciation rates, and volatilities of stock and liability may be different. Boyle and Draviam's study [29] is an interesting example of regime-switching modelling applied in option pricing achieved by [29], followed by Elliott and Siu [30], who have embedded the regime-switching modelling into the bond valuation, the concept of which has been put forward in the portfolio selection problem by Zhou and Yin [31], Chen et al. [32], and Chen and Yang [33]. The research studied in $[32,33]$ involves both the asset-liability feature and Markovian regime-switching modelling. As we all know, the models with only risk assets are valuable to be studied. Yao et al. [8] were working on the research of the continuous-time mean-variance model for only risky assets. It is rare for risk-free asset in reality; as a matter of fact, a relatively long investment is considered, corresponding to the stochastic nature real interest rates and the inflation risk (see Viceira [34]). The previous method of a nominal riskfree asset incorporated into the market will simplify the process of selecting portfolio but degenerate the GMV strategy to a bank deposit strategy with zero risks, which is not favourable to investors. Besides, the empirical evidence in the study by DeMiguel et al. [35] shows that the static global minimum-variance (GMV) strategy with only risky assets (derived by Markowitz [1]) tends to be better in performance out-of-sample among all estimated optimal strategies. Then in general, the properties of the time-consistent
MV strategies have been shown in a market only with risky assets by Chi [36] on the analysis of Yao et al. [8] and Zhang et al. [37].

In this paper, on the basis of the work of Björk et al. [3], it is determinate to make a further realistic financial model, and it makes sense to select a regime-switching market with only risky assets. Afterwards, the general expansion of the HJB equation will be reached according to the control theory with time inconsistency by Björk and Murgoci [38]. Finally, it proceeds the numerical illustrations to show our extended results and state the relationships with previous researches. The rest of the paper is completed as follows: the setting of the financial market will be explained in Section 2, with the developed structure of mean-variance asset-liability management with state-dependent risk aversion in a regime-switching market with only risky assets. Also, the $\mathrm{HJB}$ equation is generalized to the general situation. In Section 3, three different cases with derived solutions will be illustrated in details. More numerical examples are presented in Section 4 with corresponding figures and illustrations, and a conclusion is given in Section 5 .

\section{Model Formulation}

In a given probability space filtered, $\left(\Omega, \mathbf{P}, \mathscr{F},\left\{\mathfrak{J}_{t}\right\}_{0 \leq t \leq T}\right)$, let $W(t)=\left(W_{1}(t), W_{2}(t), \cdots, W_{m}(t)\right)^{\prime}$ be a standard $m$ -dimensional Brownian motion with definition of $(\Omega, \mathbf{P}, \mathscr{F}$ ) over the period of $[0, T]$. Since the involution of individual investments has been found, a few number of investors will not make much effect on the whole market. The mode of the market dynamics is described by a Markov chain process $\alpha(t)$. For that sense, the processes of $W(t)$ and $\alpha(t)$ are independent of each other. $\mathfrak{\Im}_{t}=\sigma\{W(s), \alpha(s) ; 0 \leq s \leq t\}$ could be augmented in the case of all the $\mathbf{P}$-null sets in $\mathscr{F}$, where $\mathscr{F}=\mathfrak{\Im}_{T}$. Some finite $T$ is used to denote the range of investment time. All random variables taken into consideration, in this paper, are defined within this filtered probability space. Assuming that there are $d$ regimes for the market state, it means that the Markov chain $\alpha_{t}$ gets one of the values from the set of $\{1,2,3, \cdots, d\}$ every time. By assumption, a generator $Q=\left(q_{k j}\right)_{d \times d}$ in the Markov chain with the stationary transition probabilities such that $p_{k j}(t)=\mathbb{P}(\alpha(s+t)=j \mid \alpha(s$ )$=k)$, where $s, t \geq 0, k, j=1,2, \cdots, d, \quad q_{k j}=\left.(d / d t) p_{k j}(t)\right|_{t=0}$ and $\sum_{j=1}^{d} q_{k j}=0, q_{j j}=-\sum_{j=1}^{d} q_{k j}<0, q_{k j}>0$. A financial market with continuous-time under the standard assumptions has been considered. Concretely speaking, the market assumptions in this paper are listed here with permission for continuous trading, no transaction cost or tax in trading, and infinitely divisible assets.

\subsection{Financial Market}

2.1.1. Assets. Suppose that an investor decides to allocate his wealth among $n+1$ risky assets. The prices of these risky assets meet the following requirements of stochastic differential equations (SDE) driven by the geometric Brownion motion (GBM) (1): 


$$
\left\{\begin{array}{l}
d P_{i}(t)=P_{i}(t)\left(b_{i}(t, \alpha(t)) d t+\sum_{h=1}^{m} \sigma_{i h}(t, \alpha(t)) d W_{h}(t)\right), \quad 0 \leq t \leq T, \\
P_{i}(0)=p_{i}>0, \quad i=0,1,2, \cdots, n,
\end{array}\right.
$$

where $P_{i}(t)$ means the initial prices of the risky assets, $\left(p_{i}, i=\right.$ $0,1,2, \cdots, n) ; \alpha(t)$ is defined as volatility factor; and $\left(b_{0}(t, \alpha(t\right.$ )$\left.), b_{1}(t, \alpha(t)), \cdots, b_{n}(t, \alpha(t))\right)$ and $\left(\sigma_{i h}(t, \alpha(t))\right)_{(n+1) \times m}$ refer to the appreciation rate vector and the volatility matrix of these assets, respectively, with assumption of positive continuous bounded deterministic functions of time $t$. As mentioned above, the GBM vector $\left\{W(t)=\left(W_{1}(t), W_{2}(t), \cdots, W_{m}(t)\right)^{\prime}\right\}$ is supposed to have a detailed description of all the random factors influencing the prices of risky assets.
2.1.2. Wealth Process. It is assumed that an investor endowed with an initial wealth $X_{0}$ at time 0 is intended to invest in the market dynamically through the period of $[0, T]$. Here, $X(t)$ stands for the total wealth at time $t$ for an investor, and $u_{i}(t)$ denotes the amount investment in asset $i$ and $N_{i}(t)$ for the total of units of asset $i$ in an investor's portfolio, $i=0,1,2$ $\cdots, n$. The sum of investment in the 0th asset after the deduction of liability is described as $\left\{X(t)-\sum_{i=1}^{n} u_{i}(t)\right\}$. Therefore, under the conditions above, the wealth held by the investor at time $t, X(t)$ is shown as follows:

$$
d X(t)=\sum_{i=0}^{n} N_{i}(t) d P_{i}(t)=\sum_{i=0}^{n} u_{i}(t) \frac{d P_{i}(t)}{P_{i}(t)} .
$$

To further simplify $d X(t)$ in equation (2), the SDE will be represented as

$$
\left\{\begin{array}{l}
d X(t)=\left[b_{0}(t, \alpha(t)) X(t)+B^{\prime}(t, \alpha(t)) u(t)\right] d t+\left[X(t) \sigma_{0}^{\prime}(t, \alpha(t))+u^{\prime}(t) \sigma(t, \alpha(t))\right] d W(t), \\
X(0)=X_{0} \\
\alpha(0)=k_{0}
\end{array}\right.
$$

where

$$
\left\{\begin{array}{l}
u(t)=\left(u_{1}(t), u_{2}(t), \cdots, u_{n}(t)\right)_{n \times 1}^{\prime}, \\
B(t, k)=\left(b_{1}(t, k)-b_{0}(t, k), b_{2}(t, k)-b_{0}(t, k) \cdots, b_{n}(t, k)-b_{0}(t, k)\right)_{n \times 1}^{\prime}, \\
\sigma_{i}(t, k)=\left(\sigma_{i 1}(t, k), \sigma_{i 2}(t, k), \cdots, \sigma_{i m}(t, k)\right)_{m \times 1}^{\prime}, \\
\sigma(t, k)=\left(\sigma_{1}(t, k)-\sigma_{0}(t, k), \sigma_{2}(t, k)-\sigma_{0}(t, k), \cdots, \sigma_{n}(t, k)-\sigma_{0}(t, k)\right)_{n \times m}^{\prime} .
\end{array}\right.
$$

We assume that all the functions are measurable and uniformly bounded in $[0, T]$. Here, $L_{\mathscr{F}}^{2}\left(t, T ; \mathbb{R}^{n+1}\right)$ is denoted as the set of all $\mathbb{R}^{n+1}$-valued and measurable stochastic processes $f(s, \alpha(s))$ are adjusted to $\left\{\mathfrak{J}_{s}\right\}_{s \geq t}$ on $[0, T]$ such that

$$
E\left[\int_{t}^{T}|f(s, \alpha(s))|^{2} d s\right]<+\infty .
$$

2.1.3. Liability Process. In fact, the investor in the financial market is exposed to the uncontrollable liability, with value process by the following SDE:

$$
\left\{\begin{array}{l}
d L(t)=\mu(t, \alpha(t)) d t+\rho^{\prime}(t, \alpha(t)) d W(t), \\
L(0)=l_{0}
\end{array}\right.
$$

where $L(t)$ is the stochastic liability process and $l_{0}$ is defined as the initial value of the liability. Besides, $\mu(t, \alpha(t))$ and $\rho($ $t, \alpha(t))=\left(\rho_{1}(t, \alpha(t)), \rho_{2}(t, \alpha(t)), \cdots, \rho_{m}(t, \alpha(t))\right)^{\prime} \quad$ are expressed as the appreciation and volatility in liability, respectively, on the assumption of stochastic functions at time $t$ with Markov process $\alpha(t)$. In addition, generally, the liability is functioned as the real liability excluding the random income of the investor. As a result, it turns out to be negative liabilities; the random income of the investor can be more than the real liability.

Remark 1. It is clearly to see the correlation between liability value and risky assets in the dynamic processes by $m$ -dimensional geometric Brownian motion $W(t)$. Since the investment portfolio has $n+1$ risky assets and one liability, it leads to $m \geq n+2$ in the asset liability management model. 
2.1.4. Surplus Process. Let $S(t)=X(t)-L(t)$ represent the current surplus of our fortune. By substituting the wealth and liability processes, we have

$$
\left\{\begin{array}{l}
d S(t)=\left\{b_{0}(t, \alpha(t)) S(t)-\mu(t, \alpha(t))+B^{\prime}(t, \alpha(t)) u(t)\right\} d t+\left\{S(t) \sigma_{0}^{\prime}(t, \alpha(t))-\rho^{\prime}(t, \alpha(t))+u^{\prime}(t) \sigma(t, \alpha(t))\right\} d W(t) \\
d L(t)=\mu(t, \alpha(t)) d t+\rho^{\prime}(t, \alpha(t)) d W(t) \\
S(0)=s_{0}=x_{0}-l_{0} \\
L(0)=l_{0}, \alpha(0)=k_{0}
\end{array}\right.
$$

2.2. Mean-Variance Risky Asset Management (MVRAM). First, $\mathcal{U}[0, T]$ is denoted as the set of all avaliable strategies $\left\{\left(u_{0}(t), u_{1}(t), \cdots, u_{n}(t)\right)\right\} \quad$ over $\quad[0, T]$. Naturally, the MVRAM problem will give emphasis on finding optimal admissible strategy to maximize mean-variance utility at terminal time $T$. So the objective function of $J(t, s, l, k, u)$ and the equilibrium value function of $V(t, s, l, k)$ are described mathematically as follows:

$$
\begin{gathered}
J(t, s, k, u)=E_{t, s, k}\left[S^{\mathbf{u}}(T)\right]-\frac{\gamma(s, k)}{2} \operatorname{Var}_{t, s, k}\left[S^{\mathbf{u}}(T)\right], \\
V(t, s, k)=\max _{u(\cdot) \in \mathscr{U}[0, T]}\left\{E_{t, s, k}[S(T)]-\frac{\gamma(s, k)}{2} \operatorname{Var}_{t, s, k}[S(T)]\right\} \\
=J\left(t, s, k, u^{*}\right) .
\end{gathered}
$$

where $\quad E_{t, s, k}[\cdot]=E\left[\cdot \mid S^{\mathbf{u}}(t, k)=s, L^{\mathbf{u}}(t, k)=l, \alpha^{\mathbf{u}}(t)=k\right], \quad$ in which $S^{\mathbf{u}}(t, k), L^{\mathbf{u}}(t, k), \alpha^{\mathbf{u}}(t)$ successively represent the surplus process, liability, and market dynamics obtained by using the control strategy $\mathbf{u}=\left(u_{0}(t), u_{1}(t), \cdots, u_{n}(t)\right.$, and $r($ $s, k)$ means the risk aversion coefficient depending on $s$ and $k$. As a result, $J(t, s, k, u)$ can be defined as

$$
J(t, s, k, u)=E_{t, s, k}\left[F\left(s, k, S^{\mathbf{u}}(T)\right)\right]+G\left(s, k, E_{t, s, k}\left[S^{\mathbf{u}}(T)\right]\right),
$$

where

$$
F(s, k, y)=y-\frac{\gamma(s, k)}{2} y^{2}, G(s, k, y)=\frac{\gamma(s, k)}{2} y^{2}
$$

where in here $y$ represents $E_{t, s, k}\left[S^{\mathbf{u}}(T)\right]$.

Second, let $A$ be infinitesimal generator, for any fixed $u$ $\in \mathcal{U}$; the controlled infinitesimal generator $A^{u}$ corresponded to

$$
\begin{aligned}
A^{u} W(t, s, k)= & \frac{\partial W(t, s, k)}{\partial t}+\left[b_{0} s-\mu+B^{\prime} u\right] \frac{\partial W(t, s, k)}{\partial s} \\
& +\sum_{j=1}^{d} q_{k j} W(t, s, j)+\frac{1}{2}\left\{s \sigma_{0}^{\prime}-\rho^{\prime}+u^{\prime} \sigma\right\} \\
& \cdot \frac{\partial W(t, s, k)}{\partial s^{2}}\left\{s \sigma_{0}-\rho+\sigma^{\prime} u\right\}
\end{aligned}
$$

Based on the analysis of Björk Bjrk and Murgoci [38], with the definition of equilibrium control in equation (9) and the infinitesimal generator $A^{u}$ in equation (12), the HJB equation will be extended as follow, as well as the verification of theorem.

Theorem 1 (verification theorem). It is assumed that ( $V, f$ ,$g$ ) is a solution to the following extended HJB system with the supremum of control law $\widehat{u}$ in the equation. Then, $\widehat{u}$ is subject to an equilibrium control law, and $V$ is supposed to the corresponding value function.

$$
\left\{\begin{array}{l}
\sup _{u \in \mathcal{U}}\left\{A^{u} V(t, s, k)-A^{u} f(t, s, k, s, k)+A^{u} f^{s, k}(t, s, k)-A^{u}(G \circ g)(t, s, k)+H^{u} g(t, s, k)\right\}=0, \quad 0 \leq t \leq T, \\
A^{u \wedge} f^{m, h}(t, s, k)=0, \quad 0 \leq t \leq T, \\
A^{u \wedge} g(t, s, k)=0, \quad 0 \leq t \leq T, \\
V(T, s, k)=s, \\
f^{m, h}(T, s, k)=s-\frac{\gamma(m, h)}{2} s^{2}, \\
g(T, s, k)=s,
\end{array}\right.
$$


with the following conditions and definitions:

$$
\left\{\begin{array}{l}
f^{m, h}(t, s, k)=f(t, s, k, m, h), \\
(G \circ g)(t, s, k)=G(s, k, g(t, s, k)), \\
H^{u} g(t, s, k)=G_{y}(s, k, y) \times A^{u} g(t, s, k), \\
G_{y}(s, k, y)=\frac{\partial G(s, k, y)}{\partial y}
\end{array}\right.
$$

Moreover, $f$ and $g$ have the following probabilistic representations:

$$
\begin{gathered}
f(t, s, k, m, h)=E_{t, s, k}\left[F\left(m, h, S^{u \wedge}(T)\right)\right], \\
g(t, s, k)=E_{t, s, k}\left[S^{u \wedge}(T)\right], \\
V(t, s, k)=f(t, s, k)+G(s, k, g(t, s, k)) .
\end{gathered}
$$

Proof. On the basis of the HJB equation in [2] and the objective function [3], it can be derived as

$$
V\left(t, S_{t}, k_{t}\right)=\sup _{u \in U} J\left(t, S_{t}, k_{t}, U\right)
$$

and consequently have

$$
J\left(t, S_{t}, k_{t}, U\right)=E_{t, S_{t}, k_{t}}\left[F\left(s, k, S_{T}^{U}\right)\right]+G\left(s, k, E_{t, S_{t}, k_{t}}\left[S_{T}^{U}\right]\right),
$$

where the forms of the function $F$ and function $G$ are described in (11).

When $l>t$, we have

$$
\begin{gathered}
J\left(l, S_{l}, k_{l}, U\right)=E_{l, S_{l}, k_{l}}\left[F\left(s_{l}, k_{l}, S_{T}^{U}\right)\right]+G\left(s_{l}, k_{l}, E_{l, S_{l}, k_{l}}\left[S_{T}^{U}\right]\right), \\
E_{l, S_{l}, k_{l}}\left[F\left(s_{l}, k_{l}, S_{T}^{U}\right)\right]=f^{U}\left(l, S_{l}, k_{l}, s_{l}, k_{l}\right), \\
E_{l, S_{l}, k_{l}}\left[S_{T}^{U}\right]=g^{U}\left(l, S_{l}, k_{l}\right) .
\end{gathered}
$$

Hence, equation (18) above can simply be represented as

$$
J\left(l, S_{l}, k_{l}, U\right)=f^{U}\left(l, S_{l}, k_{l}, s_{l}, k_{l}\right)+G^{U}\left(s_{l}, k_{l}, g^{U}\left(l, S_{l}, k_{l}\right)\right) .
$$

So the expectations of the equation can be shown

$$
\begin{aligned}
E_{t, S_{t}, k_{t}}\left[J\left(l, S_{l}, k_{l}, U\right)\right]= & E_{t, S_{t}, k_{t}}\left[f^{U}\left(l, S_{l}, k_{l}, s_{l}, k_{l}\right)\right. \\
& +E_{t, S_{t}, k_{t}}\left[G\left(s_{l}, k_{l}, g^{U}\left(l, S_{l}, k_{l}\right)\right)\right],
\end{aligned}
$$

and substituting this result into the definition of (17), we then have

$$
\begin{aligned}
E_{t, S_{t}, k_{t}}\left[J\left(l, S_{l}, k_{l}, U\right)\right]= & J\left(t, S_{t}, k_{t}, U\right)+E_{t, S_{t}, k_{t}} \\
& \cdot\left[f^{U}\left(l, S_{l}, k_{l}, s_{l}, k_{l}\right)\right]-E_{t, S_{t}, k_{t}}\left[F\left(s, k, S_{T}^{U}\right)\right] \\
& +E_{t, S_{t}, k_{t}}\left[G\left(s_{l}, k_{l}, g^{U}\left(l, S_{l}, k_{l}\right)\right)\right] \\
& -G\left(s, k, E_{t, S_{t}, k_{t}}\left[S_{T}^{U}\right]\right) .
\end{aligned}
$$

After the process of iteration, we obtain

$$
\begin{gathered}
\begin{aligned}
E_{t, S_{t}, k_{t}}\left[F\left(s_{l}, k_{l}, S_{T}^{U}\right)\right] & =E_{t, S_{t}, k_{t}}\left[E_{l, S_{l}, k_{l}}\left[F\left(s_{l}, k_{l}, S_{T}^{U}\right)\right]\right] \\
& =E_{t, S_{t}, k_{t}}\left[f^{U}\left(l, S_{l}, k_{l}, s_{l}, k_{l}\right)\right],
\end{aligned} \\
E_{t, S_{t}, k_{t}}\left[S_{T}^{U}\right]=E_{t, S_{t}, k_{t}}\left[E_{l, S_{l}, k_{l}} S_{T}^{U}\right]=E_{t, S_{t}, k_{t}}\left[g^{U}\left(l, S_{l}, k_{l}\right)\right] .
\end{gathered}
$$

By substituting the results of (22) and (23) back into the equation of (21), we can get

$$
\begin{aligned}
E_{t, S_{t}, k_{t}}\left[J\left(l, S_{l}, k_{l}, U\right)\right] & -E_{t, S_{t}, k_{t}}\left[f^{U}\left(l, S_{l}, k_{l}, s_{l}, k_{l}\right)\right] \\
& +E_{t, S_{t}, k_{t}}\left[f^{U}\left(l, S_{l}, k_{l}, s_{l}, k_{l}\right)\right] \\
& -J\left(t, S_{t}, k_{t}, U\right) \\
& -E_{t, S_{t}, k_{t}}\left[G\left(s_{l}, k_{l}, g^{U}\left(l, S_{l}, k_{l}\right)\right)\right] \\
& +G\left(s, k, E_{t, S_{t}, k_{t}}\left[g^{U}\left(l, S_{l}, k_{l}\right)\right]\right)=0 .
\end{aligned}
$$

Then

$$
\begin{aligned}
\sup _{u \in U} & \left\{E_{t, S_{t}, k_{t}}\left[J\left(l, S_{l}, k_{l}, U\right)\right]-E_{t, S_{t}, k_{t}}\left[f^{U}\left(l, S_{l}, k_{l}, s_{l}, k_{l}\right)\right]\right. \\
& +E_{t, S_{t}, k_{t}}\left[f^{U}\left(l, S_{l}, k_{l}, s_{l}, k_{l}\right)\right]-J\left(t, S_{t}, k_{t}, U\right) \\
& -E_{t, S_{t}, k_{t}}\left[G\left(s_{l}, k_{l}, g^{U}\left(l, S_{l}, k_{l}\right)\right)\right] \\
& \left.+G\left(s_{t}, k_{t}, E_{t, S_{t}, k_{t}}\left[g^{U}\left(l, S_{l}, k_{l}\right)\right]\right)\right\}=0 .
\end{aligned}
$$

Through our proposed problem (16) with the definition of the control law in the classic work, it can be found out that the control $U$ coincides with the equilibrium law $\widehat{u}$ in $[l, T]$, and we formulate the following results:

$$
\begin{aligned}
J\left(l, S_{l}, k_{l}, \widehat{u}\right) & =V\left(l, S_{l}, k_{l}\right), \\
f^{U}\left(l, S_{l}, k_{l}, s, k\right) & =f\left(l, S_{l}, k_{l}, s, k\right), \\
g^{U}\left(l, S_{l}, k_{l}\right) & =g\left(l, S_{l}, k_{l}\right) .
\end{aligned}
$$


Thus, the optimization problem of (25) can be solved as

$$
\begin{aligned}
\sup _{u \in U} & \left\{E_{t, S_{t}, k_{t}}\left[V\left(l, S_{l}, k_{l}\right)\right]-E_{t, S_{t}, k_{t}}\left[f\left(l, S_{l}, k_{l}, s_{l}, k_{l}\right)\right]\right. \\
+ & E_{t, S_{t}, k_{t}}\left[f\left(l, S_{l}, k_{l}, s_{l}, k_{l}\right)\right]-V\left(t, S_{t}, k_{t}\right) \\
& -E_{t, S_{t}, k_{t}}\left[G\left(s_{l}, k_{l}, g\left(l, S_{l}, k_{l}\right)\right)\right] \\
+ & \left.G\left(y_{t}, E_{t, S_{t}, k_{t}}\left[g^{U}\left(l, S_{l}, k_{l}\right)\right]\right)\right\}=0 .
\end{aligned}
$$
have

Here, by using the operator denotations of similarity, we

$$
\begin{gathered}
E_{t, S_{t}, \mathrm{k}_{t}}\left[V\left(l, S_{l}, k_{l}\right)\right]-V\left(t, S_{t}, k_{t}\right)=A^{u} V, \\
E_{t, S_{t}, k_{t}}\left[f\left(l, S_{l}, k_{l}, s_{l}, k_{l}\right)\right]=A^{u} f, \\
E_{t, S_{t}, k_{t}}\left[f\left(l, S_{l}, k_{l}\right)\right]=A^{u} f^{s, k}, \\
E_{t, S_{t}, k_{t}}\left[G\left(s_{l}, k_{l}, g\left(l, S_{l}, k_{l}\right)\right)\right]=A^{u} G, \\
G\left(s_{t}, k_{t}, E_{t, S_{t}, k_{t}}\left[g^{U}\left(l, S_{l}, k_{l}\right)\right]\right)=H^{u} g .
\end{gathered}
$$

The derivation of the extended HJB equation with stochastic volatility will be given as,

$$
\begin{aligned}
\sup _{u \in U} & \left\{A^{u} V(t, s, k)-A^{u} f(t, s, k, s, k)\right. \\
+ & A^{u} f^{s, k}(t, s, k)-A^{u}(G * g)(t, s, k) \\
+ & \left.H^{u} g(t, s, k)\right\}=0,0 \leq t \leq T .
\end{aligned}
$$

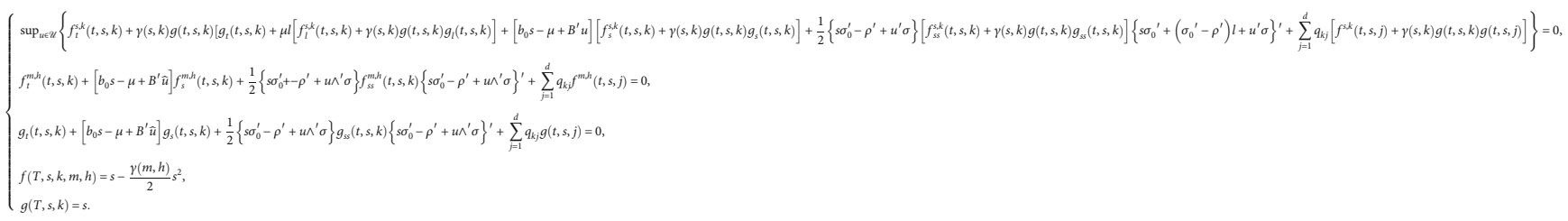

Adding up all the terms related to $u$ in (32), we have

$$
\begin{aligned}
\frac{1}{2} u^{\prime} \sigma \sigma^{\prime} u\left[f_{s s}^{s, k}+\gamma(s, k) g g_{s s}\right] \\
+\left\{\left[f_{s}^{s, k}+\gamma(s, k) g g_{s}\right] B^{\prime} u+\left[s \sigma_{0}^{\prime}-\rho^{\prime}\right]^{\prime} \sigma u\left[f_{s s}^{s, k}+\gamma(s, k) g g_{s s}\right]\right\} \\
=\frac{1}{2} \varepsilon_{1}\left\{u^{\prime} \sigma \sigma^{\prime} u+\frac{2}{\varepsilon_{1}}\left[\varepsilon_{2} B^{\prime}\left(\sigma \sigma^{\prime}\right)^{-1} \sigma+\left(s \sigma_{0}^{\prime}-\rho^{\prime}\right) \varepsilon_{1}\right] \sigma^{\prime} u\right\} \\
=\frac{1}{2} \varepsilon_{1}\left\{\sigma^{\prime} u+\frac{1}{\varepsilon_{1}}\left[\varepsilon_{2} \sigma^{\prime}\left(\sigma \sigma^{\prime}\right)^{-1} B+\left(s \sigma_{0}^{\prime}-\rho^{\prime}\right) \varepsilon_{1}\right]\right\} \\
\quad \cdot\left\{\sigma^{\prime} u+\frac{1}{\varepsilon_{1}}\left[\varepsilon_{2} B^{\prime}\left(\sigma \sigma^{\prime}\right)^{-1} \sigma+\left(s \sigma_{0}^{\prime}-\rho^{\prime}\right) \varepsilon_{1}\right]\right.
\end{aligned}
$$

\section{Solution Scheme}

3.1. The Case with a Generated $\gamma(s, k)$

Theorem 2. Under the general form of states dependent risk aversion, the optimal control strategy of MVRAM among risky assets is

$$
\widehat{u}(t, s, k)=-(\sigma \sigma)^{-1}\left\{\frac{f_{s}^{s, k}+\gamma(s, k) g g_{s}}{f_{s s}^{s, k}+\gamma(s, k) g g_{s s}} B+\left(s \sigma \sigma_{0}-\sigma \rho\right)\right\},
$$

where $f^{s, k}$ and $g$ represent the function $f^{s, k}(t, s, k)$ and $g(t, s$ ,k) by partial equations in (32).

Proof. By using the definition of the infinitesimal generator, we simplify and thus have

$$
\begin{aligned}
A^{u} V(t, s, k)-A^{u} f(t, s, k, s, k)+A^{u} f^{s, k}(t, s, k) \\
\quad-A^{u}(G \circ g)(t, s, k)+H^{u} g(t, s, k) \\
=A^{u} f^{s, k}(t, s, k)+H^{u} g(t, s, k) .
\end{aligned}
$$

The resulting extended HJB equations can be rewritten 


$$
\begin{aligned}
\widehat{u}(t, s, k) & =-\left(\sigma \sigma^{\prime}\right)^{-1}\left[\frac{\varepsilon_{2}}{\varepsilon_{1}} B+\left(s \sigma \sigma_{0}-\sigma \rho\right)\right] \\
& =-\left(\sigma \sigma^{\prime}\right)^{-1}\left\{\frac{f_{s}^{s, k}+\gamma(s, k) g g_{s}}{f_{s s}^{s, k}+\gamma(s, k) g g_{s s}} B+\left(s \sigma \sigma_{0}-\sigma \rho\right)\right\} .
\end{aligned}
$$

3.2. The Case with a Natural Choice $\gamma(s, k)$. Here, we have $\gamma(s, k)=\gamma(k) / s$ as a special form of $\gamma(s, k)$. Then, equation (30) has been changed into the following form:

$$
\widehat{u}(t, s, k)=-\left(\sigma \sigma^{\prime}\right)^{-1}\left\{\frac{s f_{s}^{s, k}+\gamma(k) g g_{s}}{s f_{s s}^{s, k}+\gamma(k) g g_{s s}} B+\left(s \sigma \sigma_{0}-\sigma \rho\right)\right\} .
$$
$(s, k)$ :

Then, we make use of the following natural choice of $\gamma$

$$
\begin{gathered}
g(t, s, k)=a(t, k) s+n(t, k), \\
f(t, s, k, m, h)=a(t, k) s+n(t, k)-\frac{\gamma(h)}{2 m} \\
\cdot\left[A(t, k) s^{2}+2 D(t, k) s+N(t, k)\right] .
\end{gathered}
$$

By differentiation, we have

$$
\begin{gathered}
g_{t}=\dot{a} s+\dot{n}, g_{s}=a, g_{s s}=0, \\
f_{t}=\dot{a} s+\dot{n}-\frac{\gamma(h)}{2 m}\left[\dot{A} s^{2}+2 \dot{D} s+\dot{N}\right],
\end{gathered}
$$

$$
f_{s}=a-\frac{\gamma(h)}{m}[A s+D], f_{s s}=-\frac{\gamma(h)}{m} A .
$$

Substituting the above expressions into $\widehat{u}(t, s, k)$, we have

$$
\widehat{u}=\mathrm{k}_{1} s+k_{2},
$$

where

$$
\begin{aligned}
& k_{1}=\left(\sigma \sigma^{-1}\left(\frac{a-\gamma(k) A+\gamma(k) a^{2}}{\gamma(k) A} B-\sigma \sigma_{0}\right),\right. \\
& k_{2}=\left(\sigma \sigma^{-1}\left[\frac{a \cdot n-D}{A} B+\sigma \rho\right] .\right.
\end{aligned}
$$

Then, we can simplify

$$
\begin{aligned}
& b_{0} s-\mu+B^{\prime} \widehat{u}=\left(b_{0}+B^{\prime} k_{1}\right) s+\left(B^{\prime} k_{2}-\mu\right)=M_{1} s+M_{2}, \\
& s \sigma_{0}-\rho+\sigma^{\prime} \widehat{u}=\left(\sigma_{0}+\sigma^{\prime} k_{1}\right) s+\left(\sigma^{\prime} k_{2}-\rho\right)=H_{1} s+H_{2} .
\end{aligned}
$$

By substituting these expressions into (32), we also have the following alternative expressions for $A^{u \wedge} f^{m, h}$ and $A^{u \wedge} g$ :

$$
\left\{\begin{array}{l}
A^{u n} f^{m, h}=-\frac{\gamma(h)}{2 m}\left[\dot{A}+2 A M_{1}+H_{1^{\prime}} H_{1} A+\sum_{j=1}^{d} q_{k j} A_{j}\right] s^{2}+\left[\dot{a}-\frac{\gamma(h)}{2 m} 2 \dot{D}+M_{1}\left(a-\frac{\gamma(h)}{m} D\right)+M_{2}\left(-\frac{\gamma(h)}{m} A\right)-\frac{\gamma(h)}{m} A H_{1^{\prime}} H_{2}+\sum_{j=1}^{d} q_{k j}\left(a_{j}-\frac{\gamma(h)}{2 m} 2 D_{j}\right)\right] s+\left[\dot{n}-\frac{\gamma(h)}{2 m} \dot{N}+M_{2}\left(a-\frac{\gamma(h)}{m} D\right)-\frac{\gamma(h)}{2 m} A H_{2}{ }^{\prime} H_{2}+\sum_{j=1}^{d} q_{k j}\left(n_{j}-\frac{\gamma(h)}{2 m} N_{j}\right)\right]=0, \\
A^{u n} g=\left(\dot{a}+M_{1} a+\sum_{j=1}^{d} q_{k j} a_{j}\right) s+\left(\dot{n}+M_{2} a+\sum_{j=1}^{d} q_{k j} n_{j}\right)=0 .
\end{array}\right.
$$

From equation (42), we can have the following system of ordinary differential equations (ODE):

$$
\left\{\begin{array}{l}
\dot{A}+2 M_{1} A+H_{1}{ }^{\prime} H_{1} A+\sum_{j=1}^{d} q_{k j} A_{j}=0, \\
\dot{a}-\frac{\gamma(h)}{m} \dot{D}+M_{1}\left(a-\frac{\gamma(h)}{m} D\right)-\frac{\gamma(h)}{m} M_{2} A-\frac{\gamma(h)}{m} A H_{1^{\prime}} H_{2}+\sum_{j=1}^{d} q_{k j}\left(a_{j}-\frac{\gamma(h)}{m} D_{j}\right)=0, \\
\dot{n}-\frac{\gamma(h)}{2 m} \dot{N}+M_{2}\left(a-\frac{\gamma(h)}{m} D\right)-\frac{\gamma(h)}{2 m} A H_{2^{\prime}} H_{2}+\sum_{j=1}^{d} q_{k j}\left(n_{j}-\frac{\gamma(h)}{2 m} N_{j}\right)=0, \\
\dot{a}+M_{1} a+\sum_{j=1}^{d} q_{k j} a_{j}=0, \\
\dot{n}+M_{2} a+\sum_{j=1}^{d} q_{k j} n_{j}=0 .
\end{array}\right.
$$

By simplifying and substituting the expressions of $M_{1}$, $M_{2}, H_{1}, H_{2}$, and $k_{1}, k_{2}$ into the above equation (43), we have the following system of ODEs:

$$
\left\{\begin{array}{l}
\dot{A}+\left[2\left(b_{0}-\delta_{2}\right)-\delta_{1}\right] A+\frac{\left(a+\gamma a^{2}\right)^{2} \delta_{1}}{\gamma^{2} A}+\sum_{j=1}^{d} q_{k j} A_{j}=0, \\
\dot{D}+\left(b_{0}-\delta_{2}-\delta_{1}\right) D+\frac{a^{2}(1+\gamma a) n \delta_{1}}{\gamma A}+\left(\delta_{3}-\mu\right) A+\sum_{j=1}^{d} q_{k j} D_{j}=0, \\
\dot{N}-\frac{\delta_{1}}{A}\left(a^{2} n^{2}-D^{2}\right)+2\left(\delta_{3}-\mu\right) D+\sum_{j=1}^{d} q_{k j} N_{j}=0, \\
\dot{a}+\left(b_{0}+\frac{a-\gamma A+\gamma a^{2}}{\gamma A} \delta_{1}-\delta_{2}\right) a+\sum_{j=1}^{d} q_{k j} a_{j}=0, \\
\dot{n}+\frac{a^{2} \delta_{1}}{A} n-\frac{a D}{A} \delta_{1}+\left(\delta_{3}-\mu\right) a+\sum_{j=1}^{d} q_{k j} n_{j}=0 .
\end{array}\right.
$$


with the terminal conditions

$$
A(T, k)=a(T, k)=1, D(T, k)=N(T, k)=n(T, k)=0,
$$

where

$$
\delta_{1}=B^{\prime}\left(\sigma \sigma^{\prime}\right)^{-1} B, \delta_{2}=B^{\prime}\left(\sigma \sigma^{\prime}\right)^{-1} \sigma \sigma_{0}, \delta_{3}=B^{\prime}\left(\sigma \sigma^{\prime}\right)^{-1} \sigma \rho
$$

Following the process of simplification, the equilibrium control can be represented as (39)

$$
\widehat{u}(t, s, k)=k_{1}(t, k) s+k_{2},
$$

where

$$
\begin{aligned}
& k_{1}=\left(\sigma \sigma^{\prime}\right)^{-1}\left(\frac{a-\gamma(k) A+\gamma(k) a^{2}}{\gamma(k) A} B-\sigma \sigma_{0}\right), \\
& k_{2}=\left(\sigma \sigma^{\prime}\right)^{-1}\left[\frac{a \cdot n-D}{A} B+\sigma \rho\right],
\end{aligned}
$$

and the equilibrium value function of correspondence is given as

$$
\begin{aligned}
V(t, s, k)= & {\left[\frac{\gamma(k)}{2}\left(a^{2}(t, k)-A(t, k)\right)+a(t, k)\right] s } \\
& +\frac{\gamma(k)}{2 s}\left[n^{2}(t, k)-N(t, k)\right] \\
& +[\gamma(k)(a(t, k) n(t, k)-D(t, k))+n(t, k)],
\end{aligned}
$$

where $A(\cdot), D(\cdot), N(\cdot), a(\cdot)$, and $n(\cdot)$ satisfy the ODE system in (44).

3.3. The Case without Liability. By letting $l(t)=0$, the asset liability problem will be tackled as a portfolio selection problem. We have $S(t)=X(t), V(t, s, k)=J(t, s, k, \widehat{u}(\cdot))$, and then, the portfolio optimal control $\widehat{u}$ turns to be

$$
\widehat{u}(t, x, k)=\left(\sigma \sigma^{\prime}\right)^{-1}\left[\left(\frac{a-\gamma A+\gamma a^{2}}{\gamma A} B-\sigma \sigma_{0}\right) x+\frac{B}{A}(a \cdot n-D)\right],
$$

which corresponds to equilibrium value function defined as

$$
\begin{aligned}
V(t, x, k)= & {\left[\frac{\gamma}{2}\left(a^{2}-A\right)+a\right] x+[\gamma(a \cdot n-D)+n] } \\
& +\frac{\gamma}{2 x}\left(n^{2}-N\right),
\end{aligned}
$$

where $A, a, D, n, N$ satisfy the following system of ODEs:

$$
\left\{\begin{array}{l}
\dot{A}+\left[2\left(b_{0}-\delta_{2}\right)-\delta_{1}\right] A+\frac{\left(a+\gamma a^{2}\right)^{2} \delta_{1}}{\gamma^{2} A}+\sum_{j=1}^{d} q_{k j} A_{j}=0 \\
\dot{D}+\left(b_{0}-\delta_{2}-\delta_{1}\right) D+\frac{a^{2}(1+\gamma a) n \delta_{1}}{\gamma A}+\sum_{j=1}^{d} q_{k j} D_{j}=0, \\
\dot{N}+\frac{a^{2} n^{2}-D^{2}}{A} \delta_{1}+\sum_{j=1}^{d} q_{k j} N_{j}=0, \\
\dot{a}+\left(b_{0}+\frac{a-\gamma A+\gamma a^{2}}{\gamma A} \delta_{1}-\delta_{2}\right) a+\sum_{j=1}^{d} q_{k j} a_{j}=0, \\
\dot{n}+\frac{a^{2} \delta_{1}}{A} n-\frac{a D}{A} \delta_{1}+\sum_{j=1}^{d} q_{k j} n_{j}=0,
\end{array}\right.
$$

with the terminal conditions

$$
A(T, k)=a(T, k)=1, D(T, k)=N(T, k)=n(T, k)=0 .
$$

The ODEs are still complicated, which requires to make a further restriction such that the first asset is risk-free, namely, $b_{0}(t, k)=r(t), \sigma_{0}(t, k)=(0, \cdots, 0)^{\prime}$, and we have $\delta_{2}$ $=0$, nor does liability. Hence, the portfolio optimal control $\widehat{u}$ is shown as follows:

$$
\widehat{u}(t, x, k)=\left(\sigma \sigma^{\prime}\right)^{-1}\left[\left(\frac{a-\gamma A+\gamma a^{2}}{\gamma A} B x+\frac{B}{A}(a \cdot n-D)\right] .\right.
$$

Then, it comes to the equilibrium value function as $V(t, x, k)=\left[\frac{\gamma}{2}\left(a^{2}-A\right)+a\right] x+[\gamma(a \cdot n-D)+n]+\frac{\gamma}{2 x}\left(n^{2}-N\right)$,

where $A, a, D, n, N$ satisfy the following system of ODEs:

$$
\left\{\begin{array}{l}
\dot{A}+\left[2 r-\delta_{1}\right] A+\frac{\left(a+\gamma a^{2}\right)^{2} \delta_{1}}{\gamma^{2} A}+\sum_{j=1}^{d} q_{k j} A_{j}=0, \\
\dot{D}+\left(r-\delta_{1}\right) D+\frac{a^{2}(1+\gamma a) n \delta_{1}}{\gamma A}+\sum_{j=1}^{d} q_{k j} D_{j}=0, \\
\dot{N}+\frac{a^{2} n^{2}-D^{2}}{A} \delta_{1}+\sum_{j=1}^{d} q_{k j} N_{j}=0, \\
\dot{a}+\left(r+\frac{a-\gamma A+\gamma a^{2}}{\gamma A} \delta_{1}\right) a+\sum_{j=1}^{d} q_{k j} a_{j}=0, \\
\dot{n}+\frac{a^{2} \delta_{1}}{A} n-\frac{a D}{A} \delta_{1}+\sum_{j=1}^{d} q_{k j} n_{j}=0,
\end{array}\right.
$$


with the terminal conditions

$$
A(T, k)=a(T, k)=1, D(T, k)=N(T, k)=n(T, k)=0 .
$$

\section{Numerical Example}

According to the research studied by Chen et al. [32] and Wei et al. [15], the market state is either "Bullish" or "Bearish" with the assumption of $d=2$, where Regime 1 refers to "Bullish" and Regime 2 to "Bearish." In this section, it is exposed to illustrate the results numerically obtained in Section 3. We present the optimal control strategy and the equilibrium value in three different situations: the first situation is state-dependent risk aversion in a regime switching market with one bond and one risky asset without liability; the second situation is different from the first with liability, while the last one is in the same situation but with two risky assets.

4.1. The Case with Liability. Similarly, all the related constant parameters in situation two can been seen in Table 1, which are specified for the illustrative purpose.

The corresponding ODE system becomes

$$
\left\{\begin{array}{l}
\dot{A}_{1}+\left[2 r_{1}-\delta_{11}\right] A_{1}+\frac{\left(a_{1}+\gamma_{1} a_{1}^{2}\right)^{2} \delta_{11}}{\gamma_{1}^{2} A_{1}}+q_{11} A_{1}+q_{12} A_{2}=0 \\
\dot{A}_{2}+\left[2 r_{2}-\delta_{12}\right] A_{2}+\frac{\left(a_{2}+\gamma_{2} a_{2}^{2}\right)^{2} \delta_{12}}{\gamma_{2}^{2} A_{2}}+q_{21} A_{1}+q_{22} A_{2}=0 \\
\dot{D}_{1}+\left(r_{1}-\delta_{11}\right) D_{1}+\left(\delta_{31}-\mu_{1}\right) A_{1}+\frac{a_{1}^{2}\left(1+\gamma_{1} a_{1}\right) n_{1} \delta_{11}}{\gamma_{1} A_{1}}+q_{11} D_{1}+q_{12} D_{2}=0 \\
\dot{D}_{2}+\left(r_{2}-\delta_{12}\right) D_{2}+\left(\delta_{32}-\mu_{2}\right) A_{2}+\frac{a_{2}^{2}\left(1+\gamma_{2} a_{2}\right) n_{2} \delta_{12}}{\gamma_{2} A_{2}}+q_{21} D_{1}+q_{22} D_{2}=0 \\
\dot{N}_{1}+2\left(\delta_{31}-\mu_{1}\right) D_{1}+\frac{a_{1}^{2} n_{1}^{2}-D_{1}^{2}}{A_{1}} \delta_{11}+q_{11} N_{1}+q_{12} N_{2}=0 \\
\dot{N}_{2}+2\left(\delta_{32}-\mu_{2}\right) D_{2}+\frac{a_{2}^{2} n_{2}^{2}-D_{2}^{2}}{A_{2}} \delta_{12}+q_{21} N_{1}+q_{22} N_{2}=0 \\
\dot{a}_{1}+\left(r_{1}+\frac{a_{1}-\gamma_{1} A_{1}+\gamma_{1} a_{1}^{2}}{\gamma_{1} A_{1}} \delta_{11}\right) a_{1}+q_{11} a_{1}+q_{12} a_{2}=0 \\
\dot{a}_{2}+\left(r_{2}+\frac{a_{2}-\gamma_{2} A_{2}+\gamma_{2} a_{2}^{2}}{\gamma_{2} A_{2}} \delta_{12}\right) a_{2}+q_{21} a_{1}+q_{22} a_{2}=0 \\
\dot{n}_{1}+\frac{a_{1}^{2} \delta_{11}}{A_{1}} n_{1}-\frac{a_{1} D_{1}}{A_{1}} \delta_{11}+\left(\delta_{31}-\mu_{1}\right) a_{1}+q_{11} n_{1}+q_{12} n_{2}=0 \\
\dot{n}_{2}+\frac{a_{2}^{2} \delta_{12}}{A_{2}} n_{2}-\frac{a_{2} D_{2}}{A_{2}} \delta_{12}+\left(\delta_{32}-\mu_{2}\right) a_{2}+q_{21} n_{1}+q_{22} n_{2}=0
\end{array}\right.
$$

with the terminal conditions

$$
\begin{gathered}
A_{1}(T)=A_{2}(T)=a_{1}(T)=a_{2}(T)=1, \\
D_{1}(T)=D_{2}(T)=N_{1}(T)=N_{2}(T)=n_{1}(T)=n_{2}(T)=0 .
\end{gathered}
$$

TABLE 1: All the related constant parameters in the case with liability.

\begin{tabular}{lcc}
\hline Parameter (symbol) & $\begin{array}{c}\text { Regime } 1 \\
(i=1)\end{array}$ & $\begin{array}{c}\text { Regime 2 } \\
(i=2)\end{array}$ \\
\hline Exit time $\left(T_{i}\right)$ & 10 & 10 \\
Risk-free interest rate $\left(r_{i}\right)$ & 0.04 & 0.04 \\
Risky asset appreciation rate $\left(b_{i}\right)$ & 0.2 & 0.05 \\
Risky asset volatility $\left(\sigma_{i}\right)$ & 0.3 & 0.07 \\
Liability appreciation rate $\left(\mu_{i}\right)$ & 0.08 & 0.04 \\
Liability volatility $\left(\rho_{i}\right)$ & 0.3 & 0.1 \\
Differentiation of transition & 0.3 & 0.7 \\
probabilities $\left(q_{i}\right)$ & 0.5 & 0.9 \\
Risk aversion $\left(\gamma_{i}\right)$ & & \\
\hline
\end{tabular}

where

$$
\delta_{11}=\frac{\left(b_{1}-r_{1}\right)^{2}}{\sigma_{1}^{2}}, \delta_{12}=\frac{\left(b_{2}-r_{2}\right)^{2}}{\sigma_{2}^{2}} \text {, }
$$

$$
\delta_{31}=\frac{\left(b_{1}-r_{1}\right) \rho_{1}}{\sigma_{1}}, \delta_{32}=\frac{\left(b_{2}-r_{2}\right) \rho_{2}}{\sigma_{2}} .
$$

4.2. The Case with a Natural Choice $\gamma(s, k)$. Again, all the related constant parameters in situation three have been represented in Table 2. All parameters are specified for the illustrative purpose.

The resulting ODE system are shown as follows:

$$
\left\{\begin{array}{l}
\dot{A}_{1}+\left[2\left(b_{01}-\delta_{21}\right)-\delta_{11}\right] A_{1}+\frac{\left(a_{1}+\gamma_{1} a_{1}^{2}\right)^{2} \delta_{11}}{\gamma_{1}^{2} A_{1}}+q_{11} A_{1}+q_{12} A_{2}=0, \\
\dot{A}_{2}+\left[2\left(b_{02}-\delta_{22}\right)-\delta_{12}\right] A_{2}+\frac{\left(a_{2}+\gamma_{2} a_{2}^{2}\right)^{2} \delta_{12}}{\gamma_{2}^{2} A_{2}}+q_{21} A_{1}+q_{22} A_{2}=0, \\
\dot{D}_{1}+\left(b_{01}-\delta_{21}-\delta_{11}\right) D_{1}+\left(\delta_{31}-\mu_{1}\right) A_{1}+\frac{a_{1}^{2}\left(1+\gamma_{1} a_{1}\right) n_{1} \delta_{11}}{\gamma_{1} A_{1}}+q_{11} D_{1}+q_{12} D_{2}=0, \\
\dot{D}_{2}+\left(b_{02}-\delta_{22}-\delta_{12}\right) D_{2}+\left(\delta_{32}-\mu_{2}\right) A_{2}+\frac{a_{2}^{2}\left(1+\gamma_{2} a_{2}\right) n_{2} \delta_{12}}{\gamma_{2} A_{2}}+q_{21} D_{1}+q_{22} D_{2}=0, \\
\dot{N}_{1}+2\left(\delta_{31}-\mu_{1}\right) D_{1}+\frac{a_{1}^{2} n_{1}^{2}-D_{1}^{2}}{A_{1}} \delta_{11}+q_{11} N_{1}+q_{12} N_{2}=0, \\
\dot{N}_{2}+2\left(\delta_{32}-\mu_{2}\right) D_{2}+\frac{a_{2}^{2} n_{2}^{2}-D_{2}^{2}}{A_{2}} \delta_{12}+q_{21} N_{1}+q_{22} N_{2}=0, \\
\dot{a}_{1}+\left(b_{01}-\delta_{21}+\frac{a_{1}-\gamma_{1} A_{1}+\gamma_{1} a_{1}^{2}}{\gamma_{1} A_{1}} \delta_{11}\right) a_{1}+q_{11} a_{1}+q_{12} a_{2}=0, \\
\dot{a}_{2}+\left(b_{02}-\delta_{22}+\frac{a_{2}-\gamma_{2} A_{2}+\gamma_{2} a_{2}^{2}}{\gamma_{2} A_{2}} \delta_{12}\right) a_{2}+q_{21} a_{1}+q_{22} a_{2}=0, \\
\dot{n}_{1}+\frac{a_{1}^{2} \delta_{11}}{A_{1}} n_{1}-\frac{a_{1} D_{1}}{A_{1}} \delta_{11}+\left(\delta_{31}-\mu_{1}\right) a_{1}+q_{11} n_{1}+q_{12} n_{2}=0, \\
\dot{n}_{2}+\frac{a_{2}^{2} \delta_{12}}{A_{2}} n_{2}-\frac{a_{2} D_{2}}{A_{2}} \delta_{12}+\left(\delta_{32}-\mu_{2}\right) a_{2}+q_{21} n_{1}+q_{22} n_{2}=0,
\end{array}\right.
$$


with the terminal conditions

$$
\begin{gathered}
A_{1}(T)=A_{2}(T)=a_{1}(T)=a_{2}(T)=1, \\
D_{1}(T)=D_{2}(T)=N_{1}(T)=N_{2}(T)=n_{1}(T)=n_{2}(T)=0 .
\end{gathered}
$$

where

$$
\begin{aligned}
& \delta_{11}=\frac{\left(b_{11}-b_{01}\right)^{2}}{\left(\sigma_{11}-\sigma_{01}\right)^{2}}, \delta_{12}=\frac{\left(b_{12}-b_{02}\right)^{2}}{\left(\sigma_{12}-\sigma_{02}\right)^{2}}, \delta_{21}=\frac{\left(b_{11}-b_{01}\right) \sigma_{01}}{\sigma_{11}-\sigma_{01}}, \\
& \delta_{22}=\frac{\left(b_{12}-b_{02}\right) \sigma_{02}}{\sigma_{12}-\sigma_{02}}, \delta_{31}=\frac{\left(b_{11}-b_{01}\right) \rho_{1}}{\sigma_{11}-\sigma_{01}}, \delta_{32}=\frac{\left(b_{12}-b_{02}\right) \rho_{2}}{\sigma_{12}-\sigma_{02}} .
\end{aligned}
$$

4.3. The Case without Liability. All the related constant parameters in situation one have been displayed in Table 3, which are specified for the illustrative purpose.

The ODE system of (56) can then be simplified in the form of the following expressions:

$$
\left\{\begin{array}{l}
\dot{A}_{1}+\left[2 r_{1}-\delta_{11}\right] A_{1}+\frac{\left(a_{1}+\gamma_{1} a_{1}^{2}\right)^{2} \delta_{11}}{\gamma_{1}^{2} A_{1}}+q_{11} A_{1}+q_{12} A_{2}=0 \\
\dot{A}_{2}+\left[2 r_{2}-\delta_{12}\right] A_{2}+\frac{\left(a_{2}+\gamma_{2} a_{2}^{2}\right)^{2} \delta_{12}}{\gamma_{2}^{2} A_{2}}+q_{21} A_{1}+q_{22} A_{2}=0 \\
\dot{D}_{1}+\left(r_{1}-\delta_{11}\right) D_{1}+\frac{a_{1}^{2}\left(1+\gamma_{1} a_{1}\right) n_{1} \delta_{11}}{\gamma_{1} A_{1}}+q_{11} D_{1}+q_{12} D_{2}=0 \\
\dot{D}_{2}+\left(r_{2}-\delta_{12}\right) D_{2}+\frac{a_{2}^{2}\left(1+\gamma_{2} a_{2}\right) n_{2} \delta_{12}}{\gamma_{2} A_{2}}+q_{21} D_{1}+q_{22} D_{2}=0 \\
\dot{N}_{1}+\frac{a_{1}^{2} n_{1}^{2}-D_{1}^{2}}{A_{1}} \delta_{11}+q_{11} N_{1}+q_{12} N_{2}=0 \\
\dot{N}_{2}+\frac{a_{2}^{2} n_{2}^{2}-D_{2}^{2}}{A_{2}} \delta_{12}+q_{21} N_{1}+q_{22} N_{2}=0 \\
\dot{a}_{1}+\left(r_{1}+\frac{a_{1}-\gamma_{1} A_{1}+\gamma_{1} a_{1}^{2}}{\gamma_{1} A_{1}} \delta_{11}\right) a_{1}+q_{11} a_{1}+q_{12} a_{2}=0 \\
\dot{a}_{2}+\left(r_{2}+\frac{a_{2}-\gamma_{2} A_{2}+\gamma_{2} a_{2}^{2}}{\gamma_{2} A_{2}} \delta_{12}\right) a_{2}+q_{21} a_{1}+q_{22} a_{2}=0 \\
\dot{n}_{2}+\frac{a_{1}^{2} \delta_{11}}{A_{1}} n_{1}-\frac{a_{1} D_{1}}{A_{1}} \delta_{11}+q_{11} n_{1}+q_{12} n_{2}=0 \\
A_{2} n_{2}-\frac{a_{2} D_{2}}{A_{2}} \delta_{12}+q_{21} n_{1}+q_{22} n_{2}=0
\end{array}\right.
$$

with the terminal conditions

$$
\begin{gathered}
A_{1}(T)=A_{2}(T)=a_{1}(T)=a_{2}(T)=1, \\
D_{1}(T)=D_{2}(T)=N_{1}(T)=N_{2}(T)=n_{1}(T)=n_{2}(T)=0 .
\end{gathered}
$$

TABLE 2: All the related constant parameters in the case with a natural choice $\gamma(s, k)$.

\begin{tabular}{lcc}
\hline Parameter (symbol) & $\begin{array}{c}\text { Regime } 1 \\
(i=1)\end{array}$ & $\begin{array}{c}\text { Regime } 2 \\
(i=2)\end{array}$ \\
\hline $\begin{array}{l}\text { Exit time }\left(T_{i}\right) \\
\text { The first risky asset appreciation rate }\end{array}$ & 0.3 & 10 \\
$\left(b_{0 i}\right)$ & 0.2 & 0.06 \\
$\begin{array}{l}\text { Other risky asset appreciation rate } \\
\left(b_{1 i}\right)\end{array}$ & 0.3 & 0.05 \\
The first risky asset volatility $\left(\sigma_{0 i}\right)$ & 0.2 & 0.09 \\
Other risky asset volatility $\left(\sigma_{1 i}\right)$ & 0.08 & 0.04 \\
Liability appreciation rate $\left(\mu_{i}\right)$ & 0.3 & 0.1 \\
Liability volatility $\left(\rho_{i}\right)$ & 0.3 & 0.7 \\
$\begin{array}{l}\text { Differentiation of transition } \\
\text { probabilities }\left(q_{i}\right)\end{array}$ & 0.5 & 0.9 \\
Risk aversion $\left(\gamma_{i}\right)$ & & \\
\hline
\end{tabular}

TABLE 3: All the related constant parameters in the case without liability.

\begin{tabular}{lcc}
\hline Parameter (symbol) & $\begin{array}{c}\text { Regime } 1 \\
(i=1)\end{array}$ & $\begin{array}{c}\text { Regime 2 } \\
(i=2)\end{array}$ \\
\hline Exit time $\left(T_{i}\right)$ & 10 & 10 \\
Risk-free interest rate $\left(r_{i}\right)$ & 0.04 & 0.04 \\
Risky asset appreciation rate $\left(b_{i}\right)$ & 0.2 & 0.05 \\
Risky asset volatility $\left(\sigma_{i}\right)$ & 0.3 & 0.07 \\
Liability appreciation rate $\left(\mu_{i}\right)$ & 0.08 & 0.04 \\
Liability volatility $\left(\rho_{i}\right)$ & 0.3 & 0.1 \\
Differentiation of transition & 0.3 & 0.7 \\
probabilities $\left(q_{i}\right)$ & 0.5 & 0.9 \\
Risk aversion $\left(\gamma_{i}\right)$ & & \\
\hline
\end{tabular}

where

$$
\delta_{11}=\frac{\left(b_{1}-r_{1}\right)^{2}}{\sigma_{1}^{2}}, \delta_{12}=\frac{\left(b_{2}-r_{2}\right)^{2}}{\sigma_{2}^{2}} .
$$

From Figures 1-6, in the "Bearish" market, we could see the optimal control strategy $u$ is increasing as time goes by along with the increase of equilibrium value. They capture the optimal strategy of the utility and keep it for the whole time, because the investor pays attention to the utility functions during the entire time. It is reasonable for the time consistent investor to sacrifice parts of the utility or happiness to secure sufficient budget in order to avoid unpredictable deficits. On the other hand, from Figures 1-6 for the "Bullish" market, the optimal control strategy is growing with time while the equilibrium value decreases. At the beginning of the investment in the "Bullish" market, the investor is confident enough to employ the strategy which would lead to a optimize equilibrium value. Over time, the investor will have less investment time to invest to maximize 


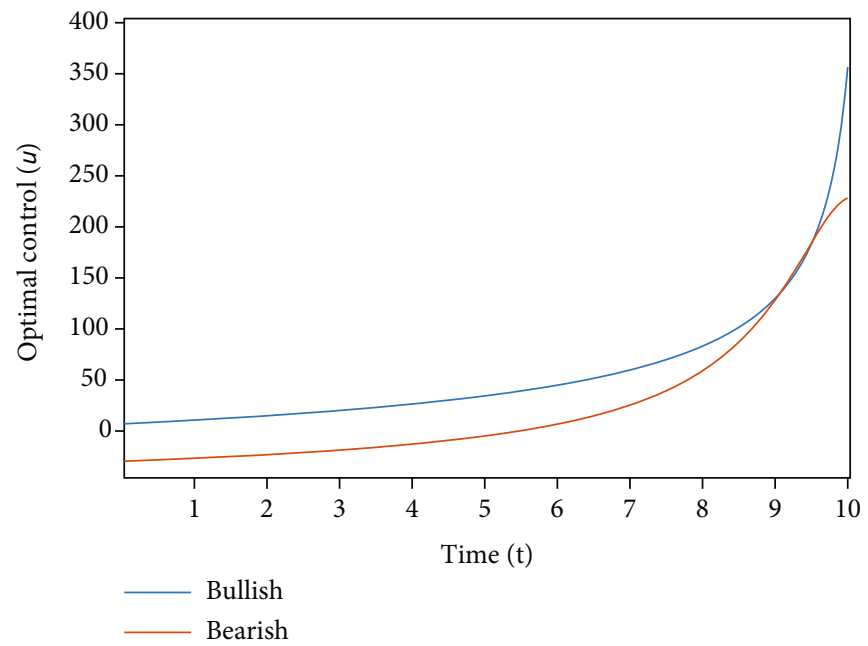

Figure 1: Optimal Control $u$.

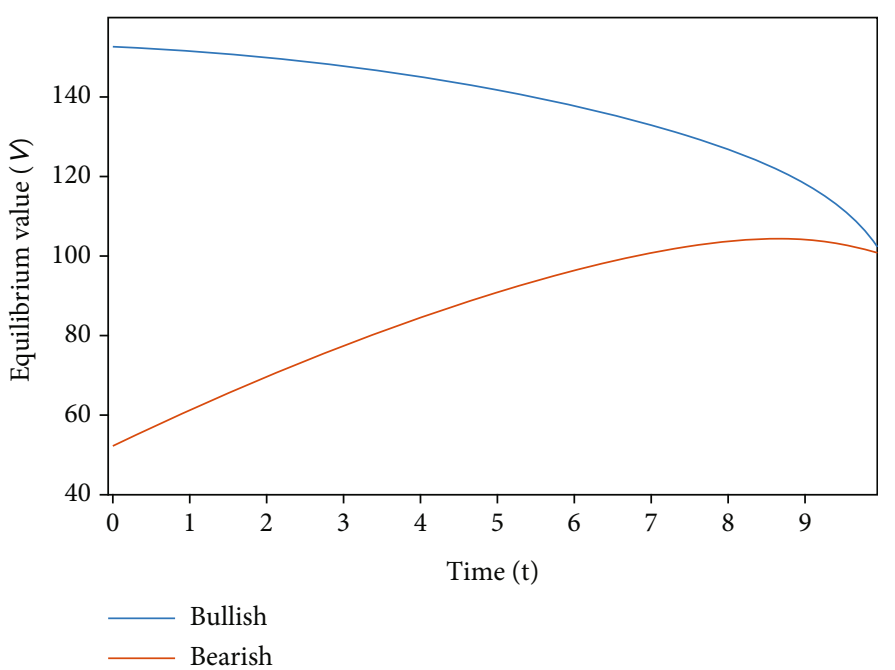

FIGURE 2: Equilibrium value $V$.

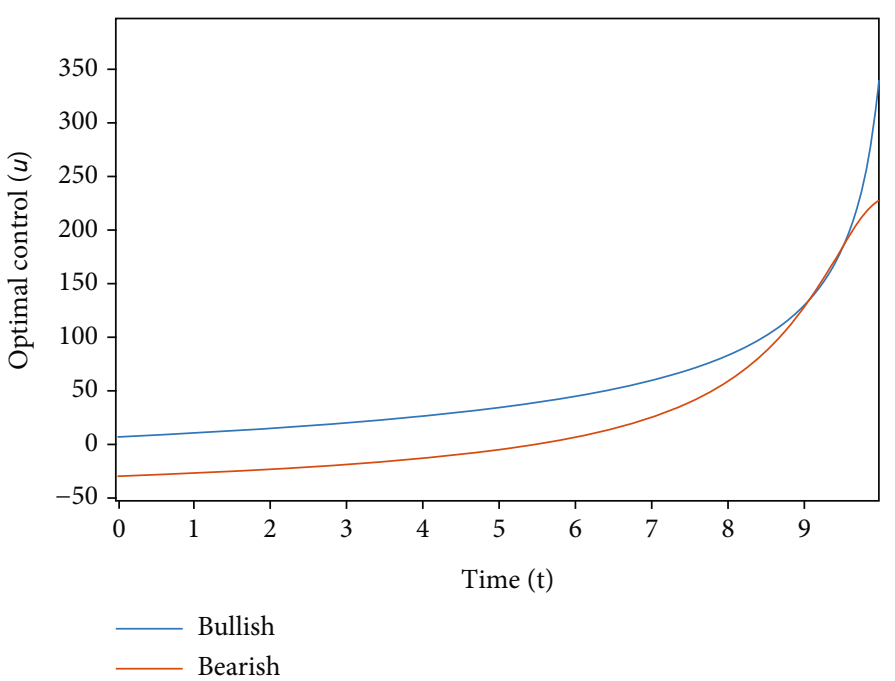

Figure 3: Optimal control $u$. 


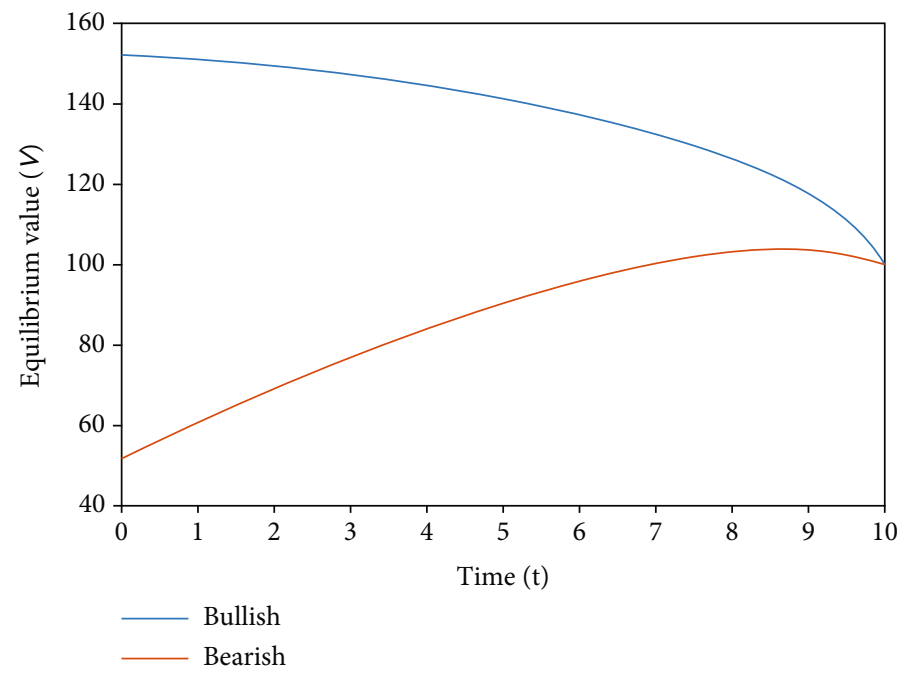

FIgURE 4: Equilibrium value $V$.

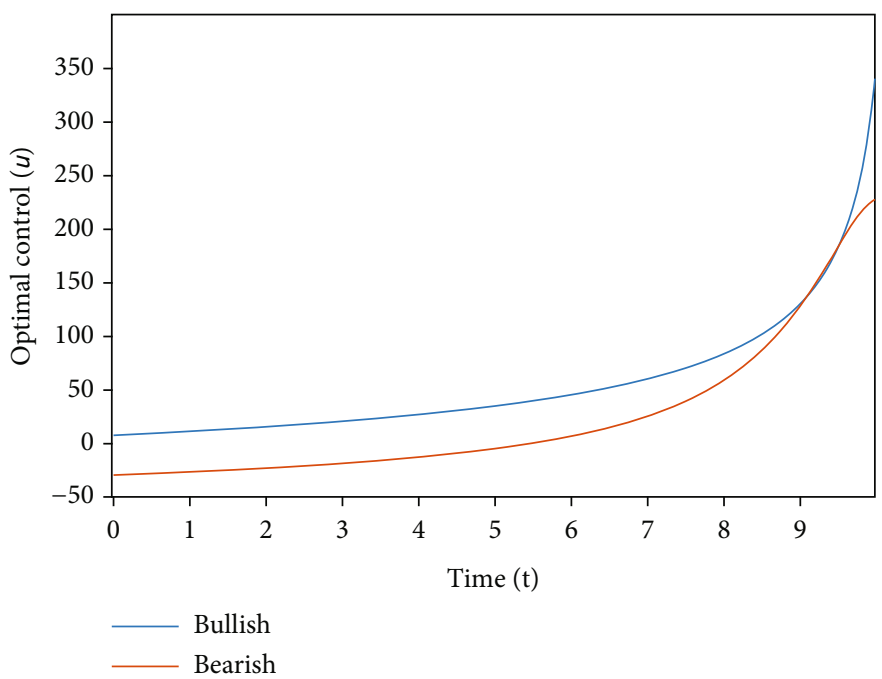

Figure 5: Optimal control $u$.

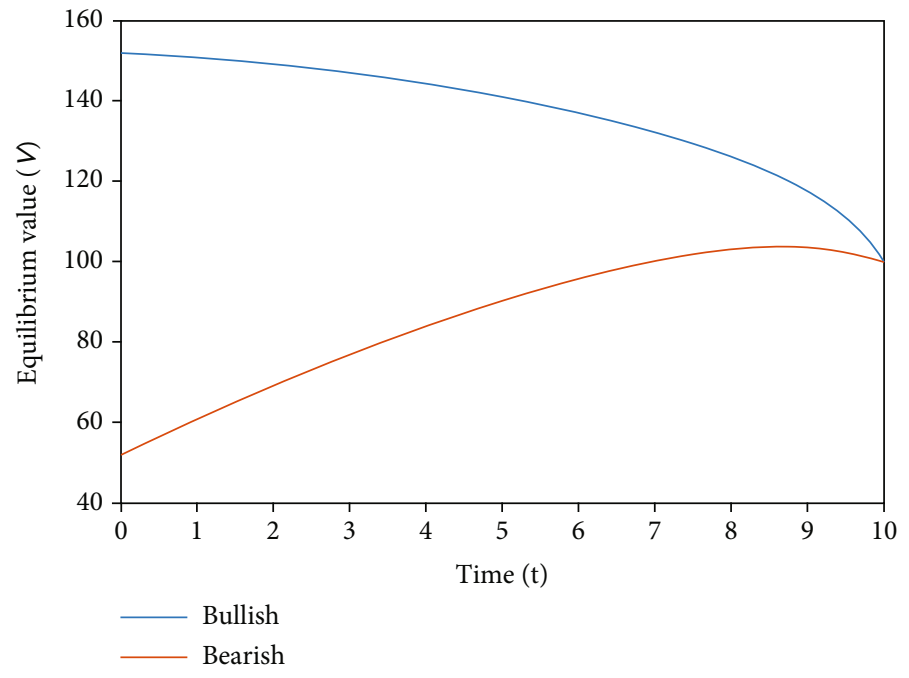

Figure 6: Equilibrium value $V$. 


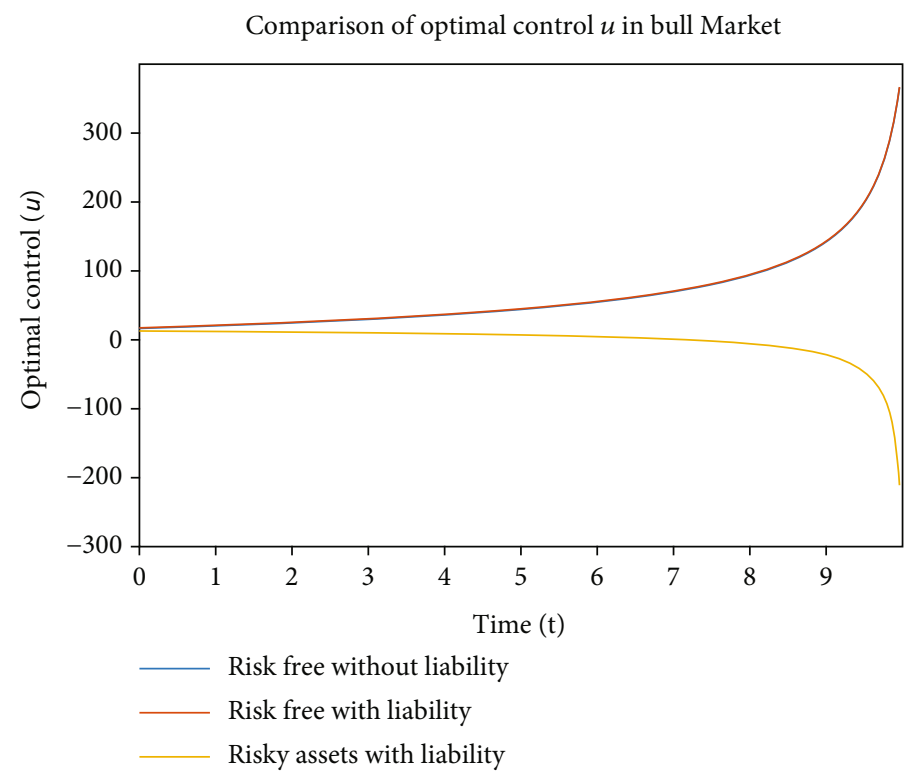

FIgURE 7: Comparison of optimal control $u$ in Bull market.

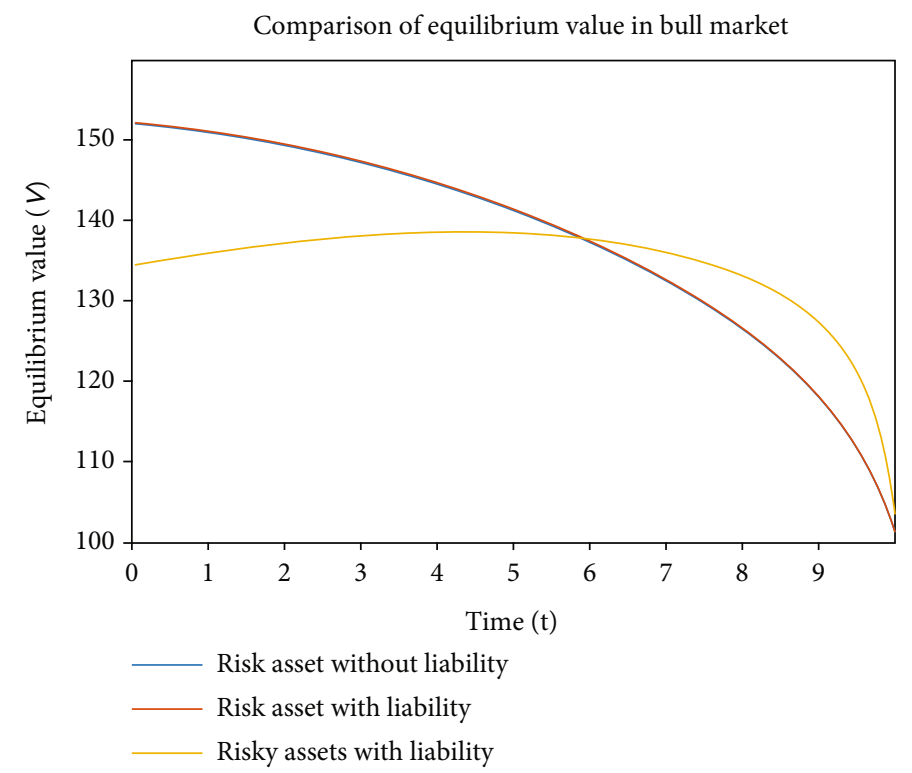

FIgURE 8: Comparison of equilibrium value in Bull market.

their current utility as well as the benefit from the "Bullish" market because of the state-dependent risk aversion.

In Figures 7 and 8, they compare the results of optimal control strategy and the equilibrium value among three different situations under the "Bullish" market.

In Figure 7, with the comparison of optimal control strategy $(u)$ in Bull market among three different situations, we find that there is no significant difference between the situations with and without liability. When the investors come across the "Bullish" market, they are willing to invest the risky asset instead of bearing the liability. However, in the situation with all risky assets, the optimal control strategy provides a way to invest in one of risky assets, which highly depends on the parameters of the two risky assets. In Figure 8, the compar- ison analysis on the equilibrium values in Bull market among three different situations has been conducted. The figure shows decreasing evidence along with levelling off, of which the reason has been explained above. For the situation with all risky assets, the equilibrium value has a more stable trend which also depends on the selected two risky assets.

In the last picture, the comparison has been made about the optimal control strategy by varying the risk aversion coefficient. The effect of the risk aversion has been analysed in Figure 9, which presents the optimal control strategy $(u)$ under three different risk aversions. Obviously, as the risk aversion increases, it can be seen that the investor is less likely to invest in risky assets, which is consistent with common sense. 


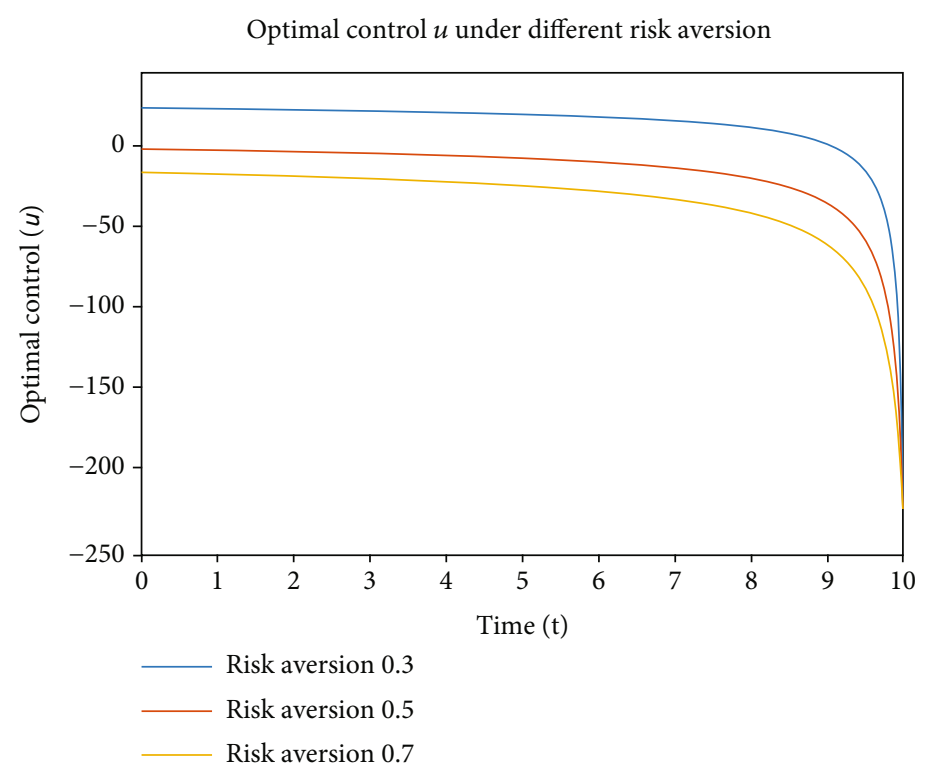

Figure 9: Optimal control $u$ under different risk aversions.

\section{Conclusions}

In this paper, the mean variance model of asset-liability management has been discussed in the case of statedependent risk aversion with only risky assets. Based on the continuous-time Markov regime-switching, this paper derives an analytical optimal control expressions theoretically in a more realistic financial market and then makes numerical analysis on a series of special cases. From the numerical results, this paper reveals the feasibility and application of introducing factors, such as regime-switching, liability, and risky asset in a mean-variance optimization framework, and also shows the relationships between a set of risk aversions and the optimal controller.

\section{Data Availability}

The data used to support the findings of this study are available from the corresponding author upon request.

\section{Conflicts of Interest}

The authors declare that there is no conflict of interests regarding the publication of this paper.

\section{Acknowledgments}

This work was supported by the National Natural Science Foundation of China under Grant 71901222 and Grant 71974204. This work was supported in part by "the Fundamental Research Funds for the Central Universities” and Zhongnan University of Economics and Law under Grant $2722020 J X 005$.

\section{References}

[1] H. Markowitz, "Portfolio selection," The Journal of Finance, vol. 7, no. 1, pp. 77-98, 1952.

[2] J. B. Detemple and F. Zapatero, "Asset prices in an exchange economy with habit formation," Econometrica, vol. 59, no. 6, pp. 1633-1657, 1991.

[3] T. Björk, A. Murgoci, and X. Y. Zhou, "Mean-variance portfolio optimization with state-dependent risk aversion," Mathematical Finance, vol. 24, no. 1, pp. 1-24, 2014.

[4] J. Yu and Y. Yuan, "Investor sentiment and the mean-variance relation," Journal of Financial Economics, vol. 100, no. 2, pp. 367-381, 2011.

[5] S. Li, C. Luong, F. Angkola, and Y. Wu, "Optimal asset portfolio with stochastic volatility under the mean-variance utility with state-dependent risk aversion," Journal of industrial and management optimization, vol. 12, no. 4, pp. 15211533, 2016.

[6] S. Li, S. Liu, Y. Zhou, Y. Wu, and X. Ge, "Optimal portfolio selection of mean-variance utility with stochastic interest rate," Journal of Function Spaces, vol. 2020, Article ID 3153297, 10 pages, 2020.

[7] V. E. Bening and V. Y. Korolev, Generalized Poisson Models and Their Applications in Insurance and Finance, VSP, International Science, Boston, 2002.

[8] H. X. Yao, Y. Z. Lai, Q. H. Ma, and H. Zheng, "Characterization of efficient frontier for mean-variance model with a drawdown constraint," Applied Mathematics and Computation, vol. 220, pp. 770-782, 2013.

[9] H. X. Yao, Y. Z. Lai, Q. H. Ma, and M. J. Jian, “Asset allocation for a DC pension fund with stochastic income and mortality risk: a multi-period mean-variance framework," Insurance: Mathematics and Economics, vol. 54, pp. 84-92, 2014.

[10] M. Leippold, F. Trojani, and P. Vanini, “A geometric approach to multiperiod mean variance optimization of assets and liabilities," Journal of Economic Dynamics and Control, vol. 28, no. 6, pp. 1079-1113, 2004. 
[11] M. C. Chiu and D. Li, "Asset and liability management under a continuous-time mean-variance optimization framework," Insurance: Mathematics and Economics, vol. 39, no. 3, pp. 330-355, 2006.

[12] D. Li and W. Ng, "Optimal dynamic portfolio selection: multiperiod mean-variance formulation," Mathematical Finance, vol. 10, no. 3, pp. 387-406, 2000.

[13] H. X. Yao, Z. Li, and S. Chen, "Continuous-time meanvariance portfolio selection with only risky assets," Economic Modelling, vol. 36, pp. 244-251, 2014.

[14] R. J. Elliott, T. K. Siu, and A. Badescu, "On mean-variance portfolio selection under a hidden Markovian regimeswitching model," Economic Modelling, vol. 27, no. 3, pp. 678-686, 2010.

[15] J. Wei, K. C. Wong, S. C. P. Yam, and S. P. Yung, "Markowitz's mean-variance asset-liability management with regime switching: a time-consistent approach," Insurance: Mathematics and Economics, vol. 53, no. 1, pp. 281-291, 2013.

[16] H. Wu and Z. Li, "Multi-period mean-variance portfolio selection with regime switching and a stochastic cash flow," Insurance: Mathematics and Economics, vol. 50, no. 3, pp. 371-384, 2012.

[17] H. L. Wu and H. Chen, "Nash equilibrium strategy for a multiperiod mean-variance portfolio selection problem with regime switching," Economic Modelling, vol. 46, pp. 79-90, 2015.

[18] J. Yu, "Optimal asset-liability management for an insurer under Markov regime switching jump-diffusion market," Asia Pacific Financial Markets, vol. 21, no. 4, pp. 317-330, 2014.

[19] Z. F. Li, Z. X. Li, S. Y. Wang, and X. T. Deng, "Optimal portfolio selection of assets with transaction costs and no short sales," International Journal of Systems Science, vol. 32, no. 5, pp. 599-607, 2001.

[20] J. Xiong and X. Y. Zhou, "Mean-variance portfolio selection under partial information," SIAM Journal on Control and Optimization, vol. 46, no. 1, pp. 156-175, 2007.

[21] C. Li and Z. Li, "Multi-period portfolio optimization for assetliability management with bankrupt control," Applied Mathematics and Computation, vol. 218, no. 22, pp. 11196-11208, 2012.

[22] A. E. B. Lim, "Quadratic hedging and mean-variance portfolio selection with random parameters in an incomplete market," Mathematics of Operations Research, vol. 29, no. 1, pp. 132161, 2004.

[23] Y. Zeng and Z. F. Li, "Asset-liability management under benchmark and mean-variance criteria in a jump diffusion market," Journal of Systems Science and Complexity, vol. 24, no. 2, pp. 317-327, 2011.

[24] A. E. B. Lim and X. Y. Zhou, "Mean-variance portfolio selection with random parameters in a complete market," Mathematics of Operations Research, vol. 27, no. 1, pp. 101-120, 2002.

[25] S. Drijver, Asset Liability Management for Pension Funds Using Multistage Mixedinteger Stochastic Programming, Laybyrint Publications, Amsterdam, 2005.

[26] P. Hilli, M. Koivu, T. Pennanen, and A. Ranne, "A stochastic programming model for asset liability management of a Finnish pension company," Annals of Operations Research, vol. 152, no. 1, pp. 115-139, 2007.

[27] T. Gerstner, M. Griebel, M. Holtz, R. Goschnick, and M. Haep, "A general asset-liability management model for the efficient simulation of portfolios of life insurance policies," Insurance: Mathematics and Economics, vol. 42, no. 2, pp. 704-716, 2008.

[28] M. C. Chiu and H. Y. Wong, "Mean-variance asset-liability management: cointegrated assets and insurance liability," European Journal of Operational Research, vol. 223, no. 3, pp. 785-793, 2012.

[29] P. Boyle and T. Draviam, "Pricing exotic options under regime switching," Insurance: Mathematics and Economics, vol. 40, no. 2, pp. 267-282, 2007.

[30] R. J. Elliott and T. K. Siu, "On Markov-modulated exponential-affine bond price formulae," Applied Mathematical Finance, vol. 16, no. 1, pp. 1-15, 2009.

[31] X. Y. Zhou and G. Yin, "Markowitz's mean-variance portfolio selection with regime switching: a continuous-time model," SIAM Journal on Control and Optimization, vol. 42, no. 4, pp. 1466-1482, 2003.

[32] P. Chen, H. L. Yang, and G. Yin, "Markowitz's mean-variance asset-liability management with regime switching: a continuous-time model," Insurance: Mathematics and Economics, vol. 43, pp. 456-465, 2008.

[33] P. Chen and H. L. Yang, "Markowitz's mean-variance assetliability management with regime switching: a multi-period model," Applied Mathematical Finance, vol. 18, no. 1, pp. 29-50, 2011.

[34] L. M. Viceira, "Bond risk, bond return volatility, and the term structure of interest rates," International Journal of Forecasting, vol. 28, no. 1, pp. 97-117, 2012.

[35] V. DeMiguel, L. Garlappi, F. J. Nogales, and R. Uppal, “A generalized approach to portfolio optimization: improving performance by constraining portfolio norms," Management Science, vol. 55, no. 5, pp. 798-812, 2009.

[36] S. P. Chi, "Time-consistent mean-variance portfolio selection with only risky assets," Economic Modelling, vol. 75, pp. 281292, 2018.

[37] M. Zhang, F. Chen, and H. X. Yao, "Mean-variance portfolio selection with only risky assets under regime switching," Economic Modelling, vol. 62, pp. 35-42, 2017.

[38] T. Björk and A. Murgoci, "A theory of Markovian timeinconsistent stochastic control in discrete time," Finance and Stochastics, vol. 18, no. 3, pp. 545-592, 2014. 